\title{
A GENERALISATION OF THE DIOPHANTINE EQUATION $x^{2}+8 \cdot 7^{b}=y^{2 r}$
}

\section{Siti Hasana Sapar ${ }^{1 \mathrm{a}, \mathrm{b}}$ and Kai Siong Yow ${ }^{2 \mathrm{a}, \mathrm{b}^{*}}$}

\author{
${ }^{\mathrm{a}}$ Institute for Mathematical Research, Universiti Putra Malaysia, 43400 UPM Serdang, Selangor, MALAYSIA. \\ ${ }^{\mathrm{b}}$ Department of Mathematics, Faculty of Science, Universiti Putra Malaysia, 43400 UPM Serdang, Selangor, \\ MALAYSIA. \\ E-mail: sitihas@upm.edu.my¹, ksyow@upm.edu.my² \\ *Corresponding Author: ksyow@upm.edu.my \\ Published: $30^{\text {th }}$ Jun 2021
}

Received: $25^{\text {th }}$ Apr 2020

Accepted: $16^{\text {th }}$ Dec 2020

DOI: https://doi.org/10.22452/mjs.vol40no2.3

\begin{abstract}
We investigate the integral solutions to the Diophantine equation $x^{2}+$ $8 \cdot 7^{b}=y^{2 r}$ where $x, y, b, r \in \mathbb{Z}^{+}$. We first generalise the forms of $x$ and $y^{r}$ that satisfy the equation. We then show the general forms of non-negative integral solutions to the equation under several conditions. We also investigate some special cases in which the integral solutions exist.
\end{abstract}

Keywords: Diophantine equation, polynomial, integral solution, generator, geometric sequence.

\section{INTRODUCTION}

A Diophantine equation is a polynomial equation in which only the integer solutions are studied. It has a long and rich literature in mathematics, particularly in number theory. The study of such equations was initiated by Diophantus of Alexandria (about AD 250). $\mathrm{He}$ was also one of the first mathematicians to introduce symbolism into algebra hence making important advances in mathematical notation (Muriefah \& Bugeaud, 2006).

The fundamental problem in studying Diophantine equations is to determine how many solutions exist. Problems in Diophantine equations are usually easy to state, but rather difficult to solve. Some sophisticated mathematical tools may be required to solve some of the problems. Fermat's Last Theorem (sometimes known as Fermat's conjecture) is one of the most popular equations since 1637, where Fermat wrote a note in the margin of his copy of Arithmetica stating that the equation $x^{n}+y^{n}=z^{n}$ has no positive integral solutions for $n>2$. The first successful proof was published in the $20^{\text {th }}$ century (Wiles, 1995).

There are many integral solutions to the equation $x^{2}+y^{2}=z^{2}$ including $(x, y, z)=(3,4,5)$ and $(5,12,13)$. The Babylonians were aware that the solution $(x, y, z)=(4961,6480,8161)$ exists as early as 1500 BC. In fact, the solutions to the equation can be derived by using the theorem of Pythagoras. 
Different Diophantine equations have been studied, including the equation,

$$
x^{2}+C=y^{n}
$$

where $x, y \geq 1$ and $n \geq 3$. Results has shown that (1) has no solution when $C=1$ (Lebesgue, 1850). Lebesgue was also the first who obtain non-trivial solutions to the equation. It was proven that when $C=-1$, the only solution is $x=3$, which is also a notable case of the Catalan conjecture (Ko, 1965). Cohn then made a huge progress to the equation by completing 77 values of parameters $C$ where $1 \leq C \leq 100$ (Cohn, 1993). The cases for $C=74$ and $C=86$ were also completed a few years later (Mignotte et al., 1996).

The study of (1) was then extended by replacing $C$ by a power of a fixed prime. Cohn proved that if $C=2^{2 k+1}$, then the equation has solutions only when $n=3$. He also pointed out that when $C=$ $2^{2 k}$, the case becomes more complex (Cohn, 1992). Le provided the complete solutions to the equation $x^{2}+2^{m}=y^{n}$ with the aid of computers (Le, 1997). The solutions for the equation $x^{2}+3^{m}=y^{n}$ when $m$ is odd, and partial results when $m$ is even, were also given a year later (Arif et al., 1998). The general solution when $m$ is even was then found by Luca with one assumption that both $x$ and $y$ are coprime (Luca, 2000). The assumption where $\operatorname{gcd}(x, y)=1$ becomes a necessary condition to the equation in some particular cases when $C$ is the square of an odd prime number (Le, 2003).

Luca solved the equation $x^{2}+2^{a}$. $3^{b}=y^{n}$, where $a$ and $b$ denote nonnegative integers and $x$ and $y$ are coprime (Luca, 2002). All positive integral solutions of the equation when $n=3$ and $n=4$ are as follows:

When $n=3$ :

$$
\begin{aligned}
(x, y)= & (5,3),(11,5),(10,7),(17,7),(46,13),(35,13),(595,73),(955,97),(2681,193), \\
& (39151,1153) .
\end{aligned}
$$

$$
\begin{aligned}
& \text { When } n=4: \\
& \begin{aligned}
(x, y)=(7,3),(23,5),(7,5),(47,7),(287,17) .
\end{aligned}
\end{aligned}
$$

Luca and Togbe then found all the integral solutions to the equation $x^{2}+2^{a} \cdot 5^{b}=$ $y^{n}$ (Luca et al., 2008). The work was extended by investigating the Diophantine equation $x^{2}+2^{a} \cdot 7^{b}=y^{n}$. The integral solutions when $a=2$ and $y=2 r$ to the equation were given (Yow et al., 2013).

In this study, we investigate the integral solutions to the equation $x^{2}+8$. $7^{b}=y^{2 r}$. We first consider all possible combinations for the term $8 \cdot 7^{b}$, and solve those equations simultaneously. We generalise the integral solutions to the equation under several conditions. We then consider some special cases in which the integral solutions exist.

Given that Diophantine equations can be used to model different types of real-life problems, this generalisation is important as it could be used to generate solutions for some relevant problems in mathematical modelling. The approaches used in this generalisation may also be extended to find integer solutions of some other Diophantine equations. 


\section{A GENERALISATION}

In this section, we determine the integral solutions to the equation $x^{2}+8 \cdot 7^{b}=y^{2 r}$, where $b, r \in \mathbb{Z}^{+}$. We first have the following definition.

Definition 1. Let $b, r \in \mathbb{Z}^{+}$. The pair of integers $\left(x, y^{r}\right)$ is a generator of solutions to the equation $x^{2}+8 \cdot 7^{b}=y^{2 r}$.

We give a general form of generators of solutions to the equation $x^{2}+8 \cdot 7^{b}=y^{2 r}$, as shown in the following lemma.

Lemma 1. Let $b, r \in \mathbb{Z}^{+}$and $r>1$. The generators of solutions to the equation $x^{2}+2^{3}$. $7^{b}=y^{2 r}$ are given by

$$
\begin{aligned}
x & =2^{2-p} \cdot 7^{b-q}-2^{p-1} \cdot 7^{q} \\
y^{r} & =2^{2-p} \cdot 7^{b-q}+2^{p-1} \cdot 7^{q}
\end{aligned}
$$

or

$$
\begin{aligned}
x & =2^{2-p} \cdot 7^{q}-2^{p-1} \cdot 7^{b-q}, \\
y^{r} & =2^{2-p} \cdot 7^{q}+2^{p-1} \cdot 7^{b-q},
\end{aligned}
$$

where $0<p<3$ and $0 \leq q \leq b$.

Proof. Given that $x^{2}+2^{3} \cdot 7^{b}=y^{2 r}$, we have

$$
\begin{aligned}
y^{2 r}-x^{2} & =2^{3} \cdot 7^{b} \\
\left(y^{r}+x\right)\left(y^{r}-x\right) & =2^{3} \cdot 7^{b} \\
\left(y^{r}+x\right)\left(y^{r}-x\right) & =2^{3-p} \cdot 2^{p} \cdot 7^{b-q} \cdot 7^{q}
\end{aligned}
$$

where $0<p<3$ and $0 \leq q \leq b$.

By comparing the factors on both sides of (2) using all the possible combinations,

$$
\text { i. } \quad \begin{array}{ll}
y^{r}+x=2^{3-p} \\
y^{r}-x=2^{p} \cdot 7^{b-q} \cdot 7^{q}
\end{array}
$$

ii. $y^{r}+x=2^{p}$

$$
y^{r}-x=2^{3-p} \cdot 7^{b-q} \cdot 7^{q}
$$

Note that when $q=0$, the sets of solutions for equations (i) and (ii) are subsets of the sets of solutions for equations (iv) and (iii), respectively. Thus, there exist four valid expressions for $\left(y^{r}+x\right)$ and $\left(y^{r}-x\right)$ as follows:

iii. $y^{r}+x=2^{3-p} \cdot 7^{b-q}$

$y^{r}-x=2^{p} \cdot 7^{q}$

iv. $\quad y^{r}+x=2^{3-p \cdot 7^{q}}$

$y^{r}-x=2^{p} \cdot 7^{b-q}$

by solving equations (iii) and (iv) simultaneously, the generators of solutions to the equation are given by 
or

$$
\begin{aligned}
x & =2^{2-p} \cdot 7^{b-q}-2^{p-1} \cdot 7^{q}, \\
y^{r} & =2^{2-p} \cdot 7^{b-q}+2^{p-1} \cdot 7^{q},
\end{aligned}
$$

$$
\begin{aligned}
x & =2^{2-p} \cdot 7^{q}-2^{p-1} \cdot 7^{b-q}, \\
y^{r} & =2^{2-p} \cdot 7^{q}+2^{p-1} \cdot 7^{b-q},
\end{aligned}
$$

where $0<p<3$ and $0 \leq q \leq b$.

Since $\left(y^{r}+x\right)$ and $\left(y^{r}-x\right)$ in (2) can be expressed both in either positive or negative signs, we now give the parity of $r$ and the sign of $y$ in the equation $x^{2}+2^{3}$.

Theorem 2. Let $b, r \in \mathbb{Z}^{+}$, and $\left(y^{r}+x\right)\left(y^{r}-x\right)=2^{3} \cdot 7^{b}$. If $\left(y^{r}+x\right)$ and $\left(y^{r}-x\right)$ are both negative, then $r$ is odd and $y$ has a negative sign.

Proof. By Lemma 1, we can see that the sets of solutions for equations (i) and (ii) are subsets of the sets of solutions for equations (iv) and (iii), respectively. Hence, we now focus on (iii) and (iv) in this proof. By using (iii) in Lemma 1 , we have $\left(y^{r}+x\right)=-\left(2^{3-p} \cdot 7^{b-q}\right)$ and $\left(y^{r}-x\right)=-\left(2^{p} \cdot 7^{q}\right)$. Solve these equations simultaneously, we obtain

and

$$
\begin{aligned}
2 x & =-\left(2^{3-p} \cdot 7^{b-q}\right)+2^{p} \cdot 7^{q} \\
x & =-\left(2^{2-p} \cdot 7^{b-q}\right)+2^{p-1} \cdot 7^{q}
\end{aligned}
$$

$$
\begin{aligned}
y^{r} & =-\left(2^{3-p} \cdot 7^{b-q}\right)+\left(2^{2-p} \cdot 7^{b-q}\right)-2^{p-1} \cdot 7^{q} \\
& =-\left(2^{3-p} \cdot 7^{b-q}\right)\left(1-2^{-1}\right)-2^{p-1} \cdot 7^{q} \\
& =-\left(2^{2-p} \cdot 7^{b-q}\right)-2^{p-1} \cdot 7^{q}
\end{aligned}
$$

where $0<p<3$ and $0 \leq q \leq b$.

On the other hand, by using (iv) in Lemma 1 , we have $\left(y^{r}+x\right)=-\left(2^{3-p} \cdot 7^{q}\right)$ and $\left(y^{r}-x\right)=-\left(2^{p} \cdot 7^{b-q}\right)$. Then, we have

and

$$
\begin{aligned}
2 x & =-\left(2^{3-p} \cdot 7^{q}\right)+2^{p} \cdot 7^{b-q} \\
x & =-\left(2^{2-p} \cdot 7^{q}\right)+2^{p-1} \cdot 7^{b-q}
\end{aligned}
$$

$$
\begin{aligned}
y^{r} & =-\left(2^{3-p} \cdot 7^{q}\right)+\left(2^{2-p} \cdot 7^{q}\right)-2^{p-1} \cdot 7^{b-q} \\
& =-\left(2^{3-p} \cdot 7^{q}\right)\left(1-2^{-1}\right)-2^{p-1} \cdot 7^{b-q} \\
& =-\left(2^{2-p} \cdot 7^{q}\right)-2^{p-1} \cdot 7^{b-q}
\end{aligned}
$$

where $0<p<3$ and $0 \leq q \leq b$. is odd.

These show that the values of $y^{r}$ are negative, which implies that $y$ is negative and $r$ 
By Lemma 1 and Theorem 2, if we express both $\left(y^{r}+x\right)$ and $\left(y^{r}-x\right)$ in positive signs, all the values of $y^{r}$ are positive regardless of what the values of $r$ are. Hence, there exist solutions for $y$ in this case. Thus, we only consider cases where both $\left(y^{r}+x\right)$ and $\left(y^{r}-x\right)$ are positive hereinafter.
Recall that we have $0 \leq q \leq b$ in Lemma 1. Since each value of $q$ needs to be considered, we let $i$ be the $i^{\text {th }}$ set of non-negative integral solutions associated with each $b$. We now determine the generators of $x_{b, i}$ and $y_{b, i}^{r}$ in the following theorem.

Theorem 3. Let $b, r \in \mathbb{Z}^{+}$. The generators $\left(x_{b, i}, y_{b, i}^{r}\right)$ of solutions to the equation $x^{2}+8$. $7^{b}=y^{2 r}$ have the following forms:

when $i$ is odd,

$$
\begin{aligned}
& x_{b, i}=7^{\frac{1}{2} i-\frac{1}{2}}\left(2 \cdot 7^{b-i+1}-1\right), \\
& y_{b, i}^{r}=7^{\frac{1}{2} i-\frac{1}{2}}\left(2 \cdot 7^{b-i+1}+1\right),
\end{aligned}
$$

when $i$ is even,

$$
\begin{aligned}
& x_{b, i}=7^{\frac{1}{2} i-1}\left(7^{b-i+2}-2\right), \\
& y_{b, i}^{r}=7^{\frac{1}{2} i-1}\left(7^{b-i+2}+2\right),
\end{aligned}
$$

where $i$ is the $i^{\text {th }}$ set of non-negative integral solutions associated with each $b$.

Proof. Let $b, r \in \mathbb{Z}^{+}$. By Lemma 1, we can see that the generators for each $i$ are given by

$$
\begin{aligned}
& x_{b, i}=2^{2-p} \cdot 7^{b-q}-2^{p-1} \cdot 7^{q} \\
& y_{b, i}^{r}=2^{2-p} \cdot 7^{b-q}+2^{p-1} \cdot 7^{q}
\end{aligned}
$$

where $0<p<3$ and $0 \leq q \leq b$. It is clear that $p=1$ or $p=2$.

Based on (3) and (4), we list down the positive integral solutions by first fixing the values of $b$, in a descending order.

Suppose $b=1$. We obtain two sets of solutions, as follows:

$$
\begin{aligned}
& x_{1,1}=13 \\
& y_{1,1}^{r}=15 \\
& x_{1,2}=5 \\
& y_{1,2}^{r}=9
\end{aligned}
$$

Suppose $b=2$. We obtain three sets of solutions, as follows:

$$
\begin{aligned}
& x_{2,1}=97=13(7)+6 \\
& y_{2,1}^{r}=99=15(7)-6 \\
& x_{2,2}=47=5(7)+12 \\
& y_{2,2}^{r}=51=9(7)-12 \\
& x_{2,3}=7 \\
& y_{2,3}^{r}=21=3(7)
\end{aligned}
$$


Similarly, we have the following results for $b=\{3,4,5,6,7\}$. Due to the infinite values of $b$ and $i$, for simplicity, we omit the results for $b>7$.

Suppose $b=3$.

$$
\begin{aligned}
& x_{3,1}=685=97(7)+6=[13(7)+6](7)+6=13\left(7^{2}\right)+6(7)+6 \\
& y_{3,1}^{r}=687=99(7)-6=[15(7)-6](7)-6=15\left(7^{2}\right)-6(7)-6 \\
& x_{3,2}=341=47(7)+12=[5(7)+12](7)+12=5\left(7^{2}\right)+12(7)+12 \\
& y_{3,2}^{r}=345=51(7)-12=[9(7)-12](7)-12=9\left(7^{2}\right)-12(7)-12 \\
& x_{3,3}=91=7(7)+6(7)=13(7) \\
& y_{3,3}^{r}=105=21(7)-6(7)=15(7) \\
& x_{3,4}=35=5(7) \\
& y_{3,4}^{r}=63=9(7)
\end{aligned}
$$

Suppose $b=4$,

$$
\begin{aligned}
& x_{4,1}=4801=13\left(7^{3}\right)+6\left(7^{2}\right)+6(7)+6 \\
& y_{4,1}^{r}=4803=15\left(7^{3}\right)-6\left(7^{2}\right)-6(7)-6 \\
& x_{4,2}=2399=5\left(7^{3}\right)+12\left(7^{2}\right)+12(7)+12 \\
& y_{4,2}^{r}=2403=9\left(7^{3}\right)-12\left(7^{2}\right)-12(7)-12 \\
& x_{4,3}=679=13\left(7^{2}\right)+6(7) \\
& y_{4,3}^{r}=693=15\left(7^{2}\right)-6(7) \\
& x_{4,4}=329=5\left(7^{2}\right)+12(7) \\
& y_{4,4}^{r}=357=9\left(7^{2}\right)-12(7) \\
& x_{4,5}=49=7^{2} \\
& y_{4,5}^{r}=147=3\left(7^{2}\right)
\end{aligned}
$$

Suppose $b=5$.

$$
\begin{aligned}
& x_{5,1}=33613=13\left(7^{4}\right)+6\left(7^{3}\right)+6\left(7^{2}\right)+6(7)+6 \\
& y_{5,1}^{r}=33615=15\left(7^{4}\right)-6\left(7^{3}\right)-6\left(7^{2}\right)-6(7)-6 \\
& x_{5,2}=16805=5\left(7^{4}\right)+12\left(7^{3}\right)+12\left(7^{2}\right)+12(7)+12 \\
& y_{5,2}^{r}=16809=9\left(7^{4}\right)-12\left(7^{3}\right)-12\left(7^{2}\right)-12(7)-12 \\
& x_{5,3}=4795=13\left(7^{3}\right)+6\left(7^{2}\right)+6(7) \\
& y_{5,3}^{r}=4809=15\left(7^{3}\right)-6\left(7^{2}\right)-6(7) \\
& x_{5,4}^{r}=2387=5\left(7^{3}\right)+12\left(7^{2}\right)+12(7) \\
& y_{5,4}^{r}=2415=9\left(7^{3}\right)-12\left(7^{2}\right)-12(7) \\
& x_{5,5}=637=13\left(7^{2}\right) \\
& y_{5,5}^{r}=735=15\left(7^{2}\right) \\
& x_{5,6}=245=5\left(7^{2}\right) \\
& y_{5,6}^{r}=441=9\left(7^{2}\right)
\end{aligned}
$$

Suppose $b=6$.

$$
\begin{aligned}
& x_{6,1}=235297=13\left(7^{5}\right)+6\left(7^{4}\right)+6\left(7^{3}\right)+6\left(7^{2}\right)+6(7)+6 \\
& y_{6,1}^{r}=235299=15\left(7^{5}\right)-6\left(7^{4}\right)-6\left(7^{3}\right)-6\left(7^{2}\right)-6(7)-6 \\
& x_{6,2}=117647=5\left(7^{5}\right)+12\left(7^{4}\right)+12\left(7^{3}\right)+12\left(7^{2}\right)+12(7)+12 \\
& y_{6,2}^{r}=117651=9\left(7^{5}\right)-12\left(7^{4}\right)-12\left(7^{3}\right)-12\left(7^{2}\right)-12(7)-12 \\
& x_{6,3}=33607=13\left(7^{4}\right)+6\left(7^{3}\right)+6\left(7^{2}\right)+6(7) \\
& y_{6,3}^{r}=33621=15\left(7^{4}\right)-6\left(7^{3}\right)-6\left(7^{2}\right)-6(7)
\end{aligned}
$$




$$
\begin{aligned}
& x_{6,4}=16793=5\left(7^{4}\right)+12\left(7^{3}\right)+12\left(7^{2}\right)+12(7) \\
& y_{6,4}^{r}=16821=9\left(7^{4}\right)-12\left(7^{3}\right)-12\left(7^{2}\right)-12(7) \\
& x_{6,5}=4753=13\left(7^{3}\right)+6\left(7^{2}\right) \\
& y_{6,5}^{r}=4851=15\left(7^{3}\right)-6\left(7^{2}\right) \\
& x_{6,6}=2303=5\left(7^{3}\right)+12\left(7^{2}\right) \\
& y_{6,6}^{r}=2499=9\left(7^{3}\right)-12\left(7^{2}\right) \\
& x_{6,7}=343=7^{3} \\
& y_{6,7}^{r}=1029=3\left(7^{3}\right)
\end{aligned}
$$

Suppose $b=7$.

$$
\begin{aligned}
& x_{7,1}=1647085=13\left(7^{6}\right)+6\left(7^{5}\right)+6\left(7^{4}\right)+6\left(7^{3}\right)+6\left(7^{2}\right)+6(7)+6 \\
& y_{7,1}^{r}=1747087=15\left(7^{6}\right)-6\left(7^{5}\right)-6\left(7^{4}\right)-6\left(7^{3}\right)-6\left(7^{2}\right)-6(7)-6 \\
& x_{7,2}=823541=5\left(7^{6}\right)+12\left(7^{5}\right)+12\left(7^{4}\right)+12\left(7^{3}\right)+12\left(7^{2}\right)+12(7)+12 \\
& y_{7,2}^{r}=823545=9\left(7^{6}\right)-12\left(7^{5}\right)-12\left(7^{4}\right)-12\left(7^{3}\right)-12\left(7^{2}\right)-12(7)-12 \\
& x_{7,3}=235291=13\left(7^{5}\right)+6\left(7^{4}\right)+6\left(7^{3}\right)+6\left(7^{2}\right)+6(7) \\
& y_{7,3}^{r}=235305=15\left(7^{5}\right)-6\left(7^{4}\right)-6\left(7^{3}\right)-6\left(7^{2}\right)-6(7) \\
& x_{7,4}=117635=5\left(7^{5}\right)+12\left(7^{4}\right)+12\left(7^{3}\right)+12\left(7^{2}\right)+12(7) \\
& y_{7,4}^{r}=117663=9\left(7^{5}\right)-12\left(7^{4}\right)-12\left(7^{3}\right)-12\left(7^{2}\right)-12(7) \\
& x_{7,5}=33565=13\left(7^{4}\right)+6\left(7^{3}\right)+6\left(7^{2}\right) \\
& y_{7,5}^{r}=33663=15\left(7^{4}\right)-6\left(7^{3}\right)-6\left(7^{2}\right) \\
& x_{7,6}=16709=5\left(7^{4}\right)+12\left(7^{3}\right)+12\left(7^{2}\right) \\
& y_{7,6}^{r}=16905=9\left(7^{4}\right)-12\left(7^{3}\right)-12\left(7^{2}\right) \\
& x_{7,7}^{r}=4459=13\left(7^{3}\right) \\
& y_{7,7}^{r}=5145=15\left(7^{3}\right) \\
& x_{7,8}=1715=5\left(7^{3}\right) \\
& y_{7,8}^{r}=3087=9\left(7^{3}\right)
\end{aligned}
$$

By collecting the generators $x_{b, i}$ and $y_{b, i}^{r}$ based on the values of $i$, we have

$$
\begin{aligned}
& x_{1,1}=13 \\
& x_{2,1}=13(7)+6 \\
& x_{3,1}=13\left(7^{2}\right)+6(7)+6 \\
& x_{4,1}=13\left(7^{3}\right)+6\left(7^{2}\right)+6(7)+6 \\
& x_{5,1}=13\left(7^{4}\right)+6\left(7^{3}\right)+6\left(7^{2}\right)+6(7)+6 \\
& \vdots \\
& x_{b, 1}=13\left(7^{b-1}\right)+6\left(7^{b-2}\right)+\cdots+6(7)+6 \\
& y_{1,1}^{r}=15 \\
& y_{2,1}^{r}=15(7)-6 \\
& y_{3,1}^{r}=15\left(7^{2}\right)-6(7)-6 \\
& y_{4,1}^{r}=15\left(7^{3}\right)-6\left(7^{2}\right)-6(7)-6 \\
& y_{5,1}^{r}=15\left(7^{4}\right)-6\left(7^{3}\right)-6\left(7^{2}\right)-6(7)-6 \\
& \vdots \\
& y_{b, 1}^{r}=15\left(7^{b-1}\right)-6\left(7^{b-2}\right)-\cdots-6(7)-6
\end{aligned}
$$


For $b \geq 1$, the general forms of

and

$$
x_{b, 1}=13\left(7^{b-1}\right)+6\left(7^{b-2}\right)+\cdots+6(7)+6
$$

$$
y_{b, 1}^{r}=15\left(7^{b-1}\right)-6\left(7^{b-2}\right)-\cdots-6(7)-6
$$

are obtained by using the induction on $b$, as follows:

(a) For the base case, suppose $b=1$. The result follows since $x_{1,1}=13$.

Suppose the result is true for $b=k>1$. Let $b=k+1$. We have

$$
\begin{aligned}
x_{k+1,1} & =7 x_{k, 1}+6 \\
& =7\left(13\left(7^{k-1}\right)+6\left(7^{k-2}\right)+6\left(7^{k-3}\right)+\cdots+6(7)+6\right)+6 \\
& =13\left(7^{k}\right)+6\left(7^{k-1}\right)+6\left(7^{k-2}\right)+\cdots+6\left(7^{2}\right)+6(7)+6 \\
& =13\left(7^{(k+1)-1}\right)+6\left(7^{(k+1)-2}\right)+6\left(7^{(k+1)-3}\right)+\cdots+6(7)+6 .
\end{aligned}
$$

Therefore, the result is also true for $b=k+1$.

(b) For the base case, suppose $b=1$. The result follows since $y_{1,1}^{r}=15$.

Suppose the result is true for $b=k>1$. Let $b=k+1$. We have

$$
\begin{aligned}
y_{k+1,1}^{r} & =7 y_{k, 1}^{r}-6 \\
& =7\left(15\left(7^{k-1}\right)-6\left(7^{k-2}\right)-6\left(7^{k-3}\right)-\cdots-6(7)-6\right)-6 \\
& =15\left(7^{k}\right)-6\left(7^{k-1}\right)-6\left(7^{k-2}\right)-\cdots-6\left(7^{2}\right)-6(7)-6 \\
& =15\left(7^{(k+1)-1}\right)-6\left(7^{(k+1)-2}\right)-6\left(7^{(k+1)-3}\right)-\cdots-6(7)-6 .
\end{aligned}
$$

Therefore, the result is also true for $b=k+1$.

By using a similar method, we obtain the following general forms:

$$
\begin{aligned}
& x_{b, 2}=5\left(7^{b-1}\right)+12\left(7^{b-2}\right)+\cdots+12(7)+12 \\
& x_{b, 3}=13\left(7^{b-2}\right)+6\left(7^{b-3}\right)+\cdots+6(7) \\
& x_{b, 4}=5\left(7^{b-2}\right)+12\left(7^{b-3}\right)+\cdots+12(7) \\
& x_{b, 5}=13\left(7^{b-3}\right)+6\left(7^{b-4}\right)+\cdots+6\left(7^{2}\right) \\
& x_{b, 6}=5\left(7^{b-3}\right)+12\left(7^{b-4}\right)+\cdots+12\left(7^{2}\right) \\
& x_{b, 7}=13\left(7^{b-4}\right)+6\left(7^{b-5}\right)+\cdots+6\left(7^{3}\right) \\
& x_{b, 8}=5\left(7^{b-4}\right)+12\left(7^{b-5}\right)+\cdots+12\left(7^{3}\right) \\
& y_{b, 2}^{r}=9\left(7^{b-1}\right)-12\left(7^{b-2}\right)-\cdots-12(7)-12 \\
& y_{b, 3}^{r}=15\left(7^{b-2}\right)-6\left(7^{b-3}\right)-\cdots-6(7) \\
& y_{b, 4}^{r}=9\left(7^{b-2}\right)-12\left(7^{b-3}\right)-\cdots-12(7) \\
& y_{b, 5}^{r}=15\left(7^{b-3}\right)-6\left(7^{b-4}\right)-\cdots-6\left(7^{2}\right) \\
& y_{b, 6}^{r}=9\left(7^{b-3}\right)-12\left(7^{b-4}\right)-\cdots-12\left(7^{2}\right) \\
& y_{b, 7}^{r}=15\left(7^{b-4}\right)-6\left(7^{b-5}\right)-\cdots-6\left(7^{3}\right) \\
& y_{b, 8}^{r}=9\left(7^{b-4}\right)-12\left(7^{b-5}\right)-\cdots-12\left(7^{3}\right)
\end{aligned}
$$

Observe that the above results can be separated into two cases according to the parity of $i$. By using the induction on $i$, we now show the general forms of generators $x_{b, i}$ and $y_{b, i}^{r}$. 
Suppose $i$ is odd. Let

$$
\begin{aligned}
& x_{b, i}=13\left(7^{b-\left(\frac{1}{2} i+\frac{1}{2}\right)}\right)+6\left(7^{b-\left(\frac{1}{2} i+\frac{1}{2}\right)-1}\right)+6\left(7^{b-\left(\frac{1}{2} i+\frac{1}{2}\right)-2}\right)+\cdots+6\left(7^{\left(\frac{1}{2} i+\frac{1}{2}\right)}\right)+ \\
& 6\left(7^{\left(\frac{1}{2} i+\frac{1}{2}\right)-1}\right) .
\end{aligned}
$$

For the base case, suppose $i=1$. It is clear that the result is true. Assume that the result is true for $i=k>1$. It can be seen that

$$
\begin{aligned}
x_{b, k+2}= & 7^{-1} \cdot x_{b, k}-6\left(7^{\frac{k}{2}-\frac{1}{2}}\right)-6\left(7^{\frac{k}{2}-\frac{3}{2}}\right) \\
= & 13\left(7^{b-\left(\frac{1}{2} k+\frac{1}{2}\right)-1}\right)+6\left(7^{b-\left(\frac{1}{2} k+\frac{1}{2}\right)-2}\right)+6\left(7^{b-\left(\frac{1}{2} k+\frac{1}{2}\right)-3}\right)+\cdots+6\left(7^{\left(\frac{1}{2} k+\frac{1}{2}\right)+1}\right)+ \\
& 6\left(7^{\left(\frac{1}{2} k+\frac{1}{2}\right)}\right)+6\left(7^{\left(\frac{1}{2} k+\frac{1}{2}\right)-1}\right)+6\left(7^{\left(\frac{1}{2} k+\frac{1}{2}\right)-2}\right)-6\left(7^{\frac{k}{2}-\frac{1}{2}}\right)-6\left(7^{\frac{k}{2}-\frac{3}{2}}\right) \\
= & 13\left(7^{b-\left(\frac{1}{2} k+\frac{1}{2}\right)-1}\right)+6\left(7^{b-\left(\frac{1}{2} k+\frac{1}{2}\right)-2}\right)+6\left(7^{b-\left(\frac{1}{2} k+\frac{1}{2}\right)-3}\right)+\cdots+6\left(7^{\left(\frac{1}{2} k+\frac{1}{2}\right)+1}\right)+ \\
& 6\left(7^{\left(\frac{1}{2} k+\frac{1}{2}\right)}\right) \\
& 13\left(7^{b-\left(\frac{1}{2}(k+2)+\frac{1}{2}\right)}\right)+6\left(7^{b-\left(\frac{1}{2}(k+2)+\frac{1}{2}\right)-1}\right)+6\left(7^{b-\left(\frac{1}{2}(k+2)+\frac{1}{2}\right)-2}\right)+\cdots+ \\
= & 6\left(7^{\left(\frac{1}{2}(k+2)+\frac{1}{2}\right)}\right)+6\left(7^{\left(\frac{1}{2}(k+2)+\frac{1}{2}\right)-1}\right) .
\end{aligned}
$$

Therefore, the result is also true for $i=k+2$. Hence, we have

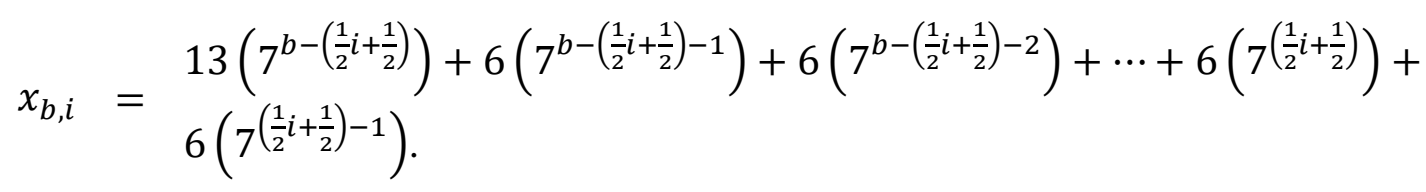

Second, let

$$
\begin{aligned}
y_{b, i}^{r}= & 15\left(7^{b-\left(\frac{1}{2} i+\frac{1}{2}\right)}\right)-6\left(7^{b-\left(\frac{1}{2} i+\frac{1}{2}\right)-1}\right)-6\left(7^{b-\left(\frac{1}{2} i+\frac{1}{2}\right)-2}\right)-\cdots-6\left(7^{\left(\frac{1}{2} i+\frac{1}{2}\right)}\right)- \\
& 6\left(7^{\left(\frac{1}{2} i+\frac{1}{2}\right)-1}\right) .
\end{aligned}
$$

For the base case, suppose $i=1$. It is clear that the result is true. Assume thathe result is true for $i=k>1$. It can be seen that

$$
\begin{aligned}
y_{b, k+2}^{r}= & 7^{-1} \cdot y_{b, k}^{r}+6\left(7^{\frac{k}{2}-\frac{1}{2}}\right)+6\left(7^{\frac{k}{2}-\frac{3}{2}}\right) \\
= & 15\left(7^{b-\left(\frac{1}{2} k+\frac{1}{2}\right)-1}\right)-6\left(7^{b-\left(\frac{1}{2} k+\frac{1}{2}\right)-2}\right)-6\left(7^{b-\left(\frac{1}{2} k+\frac{1}{2}\right)-3}\right)-\cdots-6\left(7^{\left(\frac{1}{2} k+\frac{1}{2}\right)+1}\right)- \\
& 6\left(7^{\left(\frac{1}{2} k+\frac{1}{2}\right)}\right)-6\left(7^{\left(\frac{1}{2} k+\frac{1}{2}\right)-1}\right)-6\left(7^{\left(\frac{1}{2} k+\frac{1}{2}\right)-2}\right)+6\left(7^{\frac{k}{2}-\frac{1}{2}}\right)+6\left(7^{\frac{k}{2}-\frac{3}{2}}\right) \\
= & 15\left(7^{b-\left(\frac{1}{2} k+\frac{1}{2}\right)-1}\right)-6\left(7^{b-\left(\frac{1}{2} k+\frac{1}{2}\right)-2}\right)-6\left(7^{b-\left(\frac{1}{2} k+\frac{1}{2}\right)-3}\right)-\cdots-6\left(7^{\left(\frac{1}{2} k+\frac{1}{2}\right)+1}\right)- \\
& 6\left(7^{\left(\frac{1}{2} k+\frac{1}{2}\right)}\right) \\
= & 15\left(7^{b-\left(\frac{1}{2}(k+2)+\frac{1}{2}\right)}\right)-6\left(7^{b-\left(\frac{1}{2}(k+2)+\frac{1}{2}\right)-1}\right)-6\left(7^{b-\left(\frac{1}{2}(k+2)+\frac{1}{2}\right)-2}\right)-\cdots- \\
& 6\left(7^{\left(\frac{1}{2}(k+2)+\frac{1}{2}\right)}\right)-6\left(7^{\left(\frac{1}{2}(k+2)+\frac{1}{2}\right)-1}\right) .
\end{aligned}
$$


Therefore, the result is also true for $i=k+2$. Hence, we have

$$
\begin{aligned}
y_{b, i}^{r}= & 15\left(7^{b-\left(\frac{1}{2} i+\frac{1}{2}\right)}\right)-6\left(7^{b-\left(\frac{1}{2} i+\frac{1}{2}\right)-1}\right)-6\left(7^{b-\left(\frac{1}{2} i+\frac{1}{2}\right)-2}\right)-\cdots-6\left(7^{\left(\frac{1}{2} i+\frac{1}{2}\right)}\right)- \\
& 6\left(7^{\left(\frac{1}{2} i+\frac{1}{2}\right)-1}\right) .
\end{aligned}
$$

Note that both $x_{b, i}$ and $y_{b, i}^{r}$ are geometric sequences by omitting the first term. By setting the common ratio $v=7^{-1}$, the initial value $a=7^{b-\left(\frac{1}{2} i+\frac{1}{2}\right)-1}$ and the number of terms $n=b-i$, we obtain

$$
\begin{aligned}
& x_{b, i}=13\left(7^{b-\left(\frac{1}{2} i+\frac{1}{2}\right)}\right)+6\left[\frac{7^{b-\left(\frac{1}{2} i+\frac{1}{2}\right)-1}\left(1-7^{-(b-i)}\right)}{1-7^{-1}}\right], \\
& y_{b, i}^{r}=15\left(7^{b-\left(\frac{1}{2} i+\frac{1}{2}\right)}\right)-6\left[\frac{7^{b-\left(\frac{1}{2} i+\frac{1}{2}\right)-1}\left(1-7^{-(b-i)}\right)}{1-7^{-1}}\right] .
\end{aligned}
$$

By simplifying these two equations, we have

$$
\begin{aligned}
& x_{b, i}=7^{\frac{1}{2} i-\frac{1}{2}}\left(2 \cdot 7^{b-i+1}-1\right), \\
& y_{b, i}^{r}=7^{\frac{1}{2} i-\frac{1}{2}}\left(2 \cdot 7^{b-i+1}+1\right) .
\end{aligned}
$$

Similarly, when $i$ is even, we have

$$
\begin{aligned}
& x_{b, i}=5\left(7^{b-\frac{1}{2} i}\right)+12\left(7^{b-\frac{1}{2} i-1}\right)+12\left(7^{b-\frac{1}{2} i-2}\right)+\cdots+12\left(7^{\frac{1}{2} i}\right)+12\left(7^{\frac{1}{2} i-1}\right), \\
& y_{b, i}^{r}=9\left(7^{b-\frac{1}{2} i}\right)-12\left(7^{b-\frac{1}{2} i-1}\right)-12\left(7^{b-\frac{1}{2} i-2}\right)-\cdots-12\left(7^{\frac{1}{2} i}\right)-12\left(7^{\frac{1}{2} i-1}\right) .
\end{aligned}
$$

By omitting the first term, and let $v=7^{-1}, a=7^{b-\frac{1}{2} i-1}$ and $n=b-i+1$, we obtain

$$
\begin{aligned}
& x_{b, i}=5\left(7^{b-\frac{1}{2} i}\right)+12\left[\frac{7^{b-\frac{1}{2} i-1}\left(1-7^{-(b-i+1)}\right)}{1-7^{-1}}\right], \\
& y_{b, i}^{r}=9\left(7^{b-\frac{1}{2} i}\right)-12\left[\frac{7^{b-\frac{1}{2} i-1}\left(1-7^{-(b-i+1)}\right)}{1-7^{-1}}\right] .
\end{aligned}
$$

That is,

$$
\begin{aligned}
& x_{b, i}=7^{\frac{1}{2} i-1}\left(7^{b-i+2}-2\right) \\
& y_{b, i}^{r}=7^{\frac{1}{2} i-1}\left(7^{b-i+2}+2\right)
\end{aligned}
$$

This completes the proof.

Recall that the number $i$ is the $i^{\text {th }}$ set of generators $\left(x_{b, i}, y_{b, i}^{r}\right)$ corresponds to the number of pairs of generators for solution to the equation $x^{2}+8 \cdot 7^{b}=y^{2 r}$. We now determine the range of $i$ in $x_{b, i}$ and $y_{b, i}^{r}$ for various $b$. 
Lemma 4. Let $b, r \in \mathbb{Z}^{+}$. The range of $i$ associated with each $b$ to the equation $x^{2}+8 \cdot 7^{b}=$ $y^{2 r}$ is

$$
0<i \leq b+1
$$

Proof. We consider two cases in determining the range of $i$.

First, when $i$ is odd, we have (5) from Theorem 3, that is

$$
x_{b, i}=7^{\frac{1}{2} i-\frac{1}{2}}\left(2 \cdot 7^{b-i+1}-1\right) .
$$

Since $x_{b, i}$ is non-negative, we have

$$
\begin{aligned}
2 \cdot 7^{b-i+1} & >1 \\
7^{b-i+1} & >\frac{1}{2} \\
\log 7^{b-i+1} & >\log \frac{1}{2} \\
b-i+1 & >-0.3562 \\
i & <b+1.3562 .
\end{aligned}
$$

Hence, $i<\lceil b+1.3562\rceil$. This implies that $i \leq b+1$ for all integers $b$.

Next, when $i$ is even, we consider (7) from Theorem 3, that is

$$
x_{b, i}=7^{\frac{1}{2} i-1}\left(7^{b-i+2}-2\right) .
$$

This also implies that

$$
\begin{aligned}
7^{b-i+2} & >2 \\
\log 7^{b-i+2} & >\log 2 \\
b-i+2 & >0.3562 \\
i & <b+1.6438
\end{aligned}
$$

Hence, $i<\lceil b+1.6438\rceil$. This implies that $i \leq b+1$ for all integers $b$.

By combining the two cases above, we have $0<i \leq b+1$ for all integers $b$.

From Lemma 4, we have $i \leq b+1$ for all $b$. When $b$ is even and $0<i<b+1$, we have (5) to (8) as the generators of $x_{b, i}$ and $y_{b, i}^{r}$. When $b$ is even and $i=b+1$, we show that $x_{b, i}$ and $y_{b, i}^{r}$ attain different values as in the following theorem (this can also be verified using the examples given in Theorem 3).

Theorem 5. Let $b$ be an even number and $r$ be any positive integer. Then, we have $x_{b, b+1}=$ $7^{\frac{1}{2} b}$ and $y_{b, b+1}^{r}=3 \cdot 7^{\frac{1}{2} b}$ as the generators of solutions to the equation $x^{2}+8 \cdot 7^{b}=y^{2 r}$. 
Proof. Since $i=b+1$, the parity of $i$ is odd whenever $b$ is even. By Theorem 3, we have

$$
\begin{aligned}
x_{b, i} & =7^{\frac{1}{2} i-\frac{1}{2}}\left(2 \cdot 7^{b-i+1}-1\right) \\
& =7^{\frac{1}{2}(b+1)-\frac{1}{2}}\left(2 \cdot 7^{b-(b+1)+1}-1\right) \\
& =7^{\frac{1}{2} b}(2-1) \\
& =7^{\frac{1}{2} b} .
\end{aligned}
$$

Similarly, we have

$$
\begin{aligned}
y_{b, i}^{r} & =7^{\frac{1}{2} i-\frac{1}{2}}\left(2 \cdot 7^{b-i+1}+1\right) \\
& =7^{\frac{1}{2}(b+1)-\frac{1}{2}}\left(2 \cdot 7^{b-(b+1)+1}+1\right) \\
& =3 \cdot 7^{\frac{1}{2} b} .
\end{aligned}
$$

This completes the proof.

Corollary 6. Let $b$ be an odd number and $r$ be any positive integer. Then, we have $x_{b, b+1}=$ $5 \cdot 7^{\frac{b-1}{2}}$ and $y_{b, b+1}^{r}=9 \cdot 7^{\frac{b-1}{2}}$ as the generators of solutions to the equation $x^{2}+8 \cdot 7^{b}=$ $y^{2 r}$.

Proof. By using a similar approach as in Theorem 5 (this can also be verified by using the result in Theorem 3).

\section{SOME SPECIAL CASES}

In this section, we show that when $r=2$, there exist integral solutions to the equation $x^{2}+8 \cdot 7^{b}=y^{2 r}$, for certain values of $b$ and $i$. We then show that there is no integral solution to the equation if $r \neq 2$.

Theorem 7. Let $t \in \mathbb{Z}^{+}$. Then, $x_{b, i}=5 \cdot 7^{2(t-1)}$ and $y_{b, i}=3 \cdot 7^{t-1}$ are the integral solutions to the equation $x^{2}+8 \cdot 7^{b}=y^{4}$ if and only if $b=4 t-3$ and $i=4 t-2$.

Proof. $(\Longrightarrow)$ Let $x_{b, i}$ and $y_{b, i}$ be as stated. Then, we have

$$
\begin{aligned}
\left(5 \cdot 7^{2(t-1)}\right)^{2}+8 \cdot 7^{b} & =\left(3 \cdot 7^{t-1}\right)^{4} \\
7^{b} & =7^{4 t-3} .
\end{aligned}
$$

This implies that $b=4 t-3$.

Since $x_{b, i}=5 \cdot 7^{2(t-1)}$ and $r=2$, by using (7), we have

$$
\begin{aligned}
7^{\frac{1}{2} i-1}\left(7^{b-i+2}-2\right) & =5 \cdot 7^{2(t-1)} \\
7^{4 t-\frac{1}{2} i-2}-2 \cdot 7^{\frac{1}{2} i-1} & =5 \cdot 7^{2(t-1)} \\
7^{2\left(2 t-\frac{1}{2} i\right)-1}-2 & =5 \cdot 7^{\left(2 t-\frac{1}{2} i\right)-1} .
\end{aligned}
$$

(since $b=4 t-3$ )

(multiply by $7^{-\frac{1}{2} i+1}$ ) 
Now, by letting $x=7^{2 t-\frac{1}{2} i}$ and rearranging the equation, we have

$$
\begin{aligned}
7^{-1} \cdot x^{2}-7^{-1} \cdot 5 x-2 & =0 \\
x^{2}-5 x-14 & =0 \\
(x+2)(x-7) & =0
\end{aligned}
$$

This implies that $x=-2$ or $x=7$.

When $x=-2$, we have $7^{2 t-\frac{1}{2} i}=-2$. There is an inconsistency since the term on the left-hand side should always be positive.

Secondly, when $x=7$, we have

$$
\begin{aligned}
7^{2 t-\frac{1}{2} i} & =7 \\
2 t-\frac{1}{2} i & =1 \\
i & =4 t-2 .
\end{aligned}
$$

$(\Leftarrow)$ Let $b=4 t-3$ and $i=4 t-2$. By Theorem 3, when $i$ is even, we have

and

$$
x_{b, i}=7^{\frac{1}{2} i-1}\left(7^{b-i+2}-2\right)
$$

$$
y_{b, i}^{r}=7^{\frac{1}{2} i-1}\left(7^{b-i+2}+2\right)
$$

as the generators of solutions to the equation $x^{2}+8 \cdot 7^{b}=y^{2 r}$. Let $r=2$. Then, we have $x^{2}+8 \cdot 7^{b}=y^{4}$. The generators of integral solutions for $x_{b, i}$ and $y_{b, i}^{2}$ in which $b=4 t-3$ and $i=4 t-2$ are given by

and

$$
\begin{aligned}
x_{b, i} & =x_{4 t-3,4 t-2} \\
& =7^{\frac{1}{2}(4 t-2)-1}\left(7^{(4 t-3)-(4 t-2)+2}-2\right) \\
& =7^{2 t-2}(7-2) \\
& =5 \cdot 7^{2(t-1)}
\end{aligned}
$$

$$
\begin{aligned}
y_{b, i}^{2} & =y_{4 t-3,4 t-2}^{2} \\
& =7^{\frac{1}{2}(4 t-2)-1}\left(7^{(4 t-3)-(4 t-2)+2}+2\right) \\
& =7^{2 t-2}(7+2) \\
& =3^{2} \cdot 7^{2(t-1)}
\end{aligned}
$$

Therefore, $y_{4 t-3,4 t-2}=3 \cdot 7^{t-1}$.

Corollary 8. Let $t \in \mathbb{Z}^{+}, b=4 t-3$ and $i=4 t-2$. Then, $x_{b, i}=5 \cdot 7^{2(t-1)}$ and $y_{b, i}=3$. $7^{t-1}$ are the integral solutions to the equation $x^{2}+8 \cdot 7^{b}=y^{2 r}$ if and only if $r=2$.

Theorem 9. Let $t \in \mathbb{Z}^{+}, b=4 t-3, i=4 t-2$ and $r>1$. If $r \neq 2$, the equation $x^{2}+8$. $7^{b}=y^{2 r}$ has no integral solution. 
Proof. By Theorem 3, when $i$ is even, the integral solutions to the equation $x^{2}+8 \cdot 7^{b}=y^{2 r}$ are generated by (7) and (8). Since $r>1$ and $r \neq 2$, possible values of $r$ are shown in the following table.

Table 1. Values of $r$ to the equation $x^{2}+8 \cdot 7^{b}=y^{2 r}$

Cases Values of $\mathrm{r}$

Case $1: r \equiv 0(\bmod 2) \quad r=2 s, s>1$

Case $2: r \equiv 1(\bmod 2) \quad r=1+2 s, s \geq 1$

By substituting $b$ and $i$ into (8), we have

$$
\begin{aligned}
y_{b, i}^{r} & =7^{\frac{1}{2}(4 t-2)-1}\left(7^{4 t-3-(4 t-2)+2}+2\right) \\
& =3^{2} \cdot 7^{(2 t-2)} \\
y_{b, i} & =\left(3 \cdot 7^{(t-1)}\right)^{\frac{2}{r}} .
\end{aligned}
$$

Now, substitute the two different forms of values of $r$ into (9), we have the two following cases.

Case 1: Suppose $r=2 s$.

$$
y_{b, i}=\left(3 \cdot 7^{(t-1)}\right)^{\frac{1}{s}} .
$$

Since $s>1$, the term $3^{\frac{1}{s}}$ is an irrational number. Thus, there exist no integer values for $y_{b, i}$. Hence, there is no integral solution to the equation when $r=2 s$.

Case 2: Suppose $r=1+2 s$.

$$
y_{b, i}=\left(3 \cdot 7^{(t-1)}\right)^{\frac{2}{1+2 s}} .
$$

Since $s \geq 1$, the term $3^{\frac{2}{1+2 s}}$ is an irrational number. Thus, there exist no integer values for $y_{b, i}$. It follows that there is no integral solution to the equation when $r=1+2 s$.

Therefore, we conclude that the equation $x^{2}+8 \cdot 7^{b}=y^{2 r}$ has no integral solution if $r \neq 2$, together with the stated conditions.

\section{CONCLUSIONS AND FUTURE WORK}

In this study, we give two pairs of

When $i$ is odd, we have

$$
\begin{aligned}
& x_{b, i}=7^{\frac{1}{2} i-\frac{1}{2}}\left(2 \cdot 7^{b-i+1}-1\right), \\
& y_{b, i}^{r}=7^{\frac{1}{2} i-\frac{1}{2}}\left(2 \cdot 7^{b-i+1}+1\right),
\end{aligned}
$$

and when $i$ is even, we obtain

$$
\begin{aligned}
& x_{b, i}=7^{\frac{1}{2} i-1}\left(7^{b-i+2}-2\right), \\
& y_{b, i}^{r}=7^{\frac{1}{2} i-1}\left(7^{b-i+2}+2\right),
\end{aligned}
$$

generators $\left(x_{b, i}, y_{b, i}^{r}\right)$ to the equation $x^{2}+$ $8 \cdot 7^{b}=y^{2 r}$ according to the parity of $i$. 
where $i$ is the $i^{\text {th }}$ set of non-negative integral solutions associated with each $b$. We then show that $i \in\{1,2, \ldots, b+1\}$ for all integers $b$. The generators of solutions to the equation when $i=b+1$ are also given.

We then investigate the case where $r=2$, and give the integral solutions to the equation $x^{2}+8 \cdot 7^{b}=y^{4}$ using some specific values of $b$ and $i$. We also show that there exists no integral solution to the equation when $r \neq 2$ under the same condition.

For future work, we could generalise a more general equation $x^{2}+$ $2^{a} \cdot 7^{b}=y^{n}$. Likewise, we could also focus on the cases when $n$ is odd, where $x, y, a, b \in \mathbb{Z}^{+}$.

\section{ACKNOWLEDGEMENT}

This research was partially supported by Universiti Putra Malaysia under Putra Grant GP-IPM/2020/9684300.

\section{REFERENCES}

Abu Muriefah, F. S. \& Bugeaud, Y. (2006). The Diophantine equation $x^{2}+c=y^{n}:$ a brief overview. Revista Colombiana de Matemáticas. 40: 31-37.

Arif, S. A. and Abu Muriefah, F. S. (1998). The Diophantine equation $x^{2}+3^{m}=y^{n} . \quad$ International Journal of Mathematics and Mathematical Sciences, 21(3): 619620.

Cohn, J. H. E. (1992). The Diophantine equation $x^{2}+2^{k}=y^{n}$. Arch. Math. (Basel). 59: 341-344.
Cohn, J. H. E. (1993). The Diophantine equation $\quad x^{2}+c=y^{n}$. Acta Arithmetica, 65: 367-381.

Ko, C. (1965). On the Diophantine equation $x^{2}=y^{n}+1, x y \neq 0$. Scientia Sinica, 14: 457-460.

Le, M. (1997). Diophantine equation $x^{2}+$ $2^{m}=y^{n}$. Chinese Science Bulletin, 42: 1515-1517.

Le, M. (2003). On the Diophantine equation $\quad x^{2}+p^{2}=y^{n}$. Publ. Math. Debrecen. 63: 67- 78 .

Lebesgue, V. A. (1850). Sur l'impossibilité en nombres entiers de l'équation $x^{m}=y^{2}+1$. Nouvella Annals Des Mathemetics, 78: 26-35.

Luca, F. (2000). On a Diophantine equation. Bulletin of the Australian Mathematical Society, 61: 241-246.

Luca, F. (2002). On the equation $x^{2}+$ $2^{a} \cdot 3^{b}=y^{n}$. International Journal of Mathematics and Mathematical Sciences, 29: 239244.

Luca, F. and Togbe, A. (2008). On the Diophantine equation $x^{2}+2^{a}$. $5^{b}=y^{n}$. International Journal of Number Theory, 4: 973-979.

Mignotte, M. and Weger, B. M. M. (1996). On the Diophantine equation $x^{2}+$ $74=y^{5} \quad$ and $\quad x^{2}+86=y^{5}$. Glasgow Mathematical Journal, 38(1): 77-85.

Wiles, A. J. (1995). Modular elliptic curves and Fermat's Last Theorem. Annals of Mathematics, 141(3): 443-551.

Yow, K. S., Sapar, S. H. and Atan, K. A. (2013). On the Diophantine equation $\quad x^{2}+4 \cdot 7^{b}=y^{2 r}$. Pertanika J. Sci. \& Technol, 21(2): 443-458. 